Rev Biomed 2002; 13:76-82.

\title{
Incremento de aberraciones cromosómicas e intercambio de cromátides hermanas en personas sanas con exposición laboral a rayos $X$.
}

José M. Ceballos-Quintal, Doris Pinto-Escalante, Jorge Canto-Herrera.

Laboratorio de Genética, Centro de Investigaciones Regionales “Dr. Hideyo Noguchi”, Universidad Autónoma de Yucatán, Mérida, Yucatán, México.

\section{RESUMEN.}

Introducción. Las radiaciones ionizantes producen diversos efectos en el ser humano, dependiendo de la magnitud y la fuente de exposición. Una de éstas es la exposición laboral a rayos $\mathrm{X}$, en la que puede ocurrir una exposición crónica de dosis pequeñas. La identificación de marcadores biológicos para el reconocimiento del efecto temprano a la exposición, resultan de utilidad para la evaluación de grupos en riesgo. En este trabajo analizamos las manifestaciones citogenéticas resultantes de la exposición laboral a radiaciones ionizantes $\mathrm{X}$ usadas para diagnóstico, con el propósito de determinar los cambios cromosómicos en individuos expuestos por diferentes períodos de tiempo y con diferentes grados de exposición.

Material y Métodos. Se incluyó individuos expuestos a radiación ionizante $\mathrm{X}$ y sujetos sanos no expuestos. Los análisis realizados a todos los sujetos fueron: citometría hemática completa y análisis cromosómico: aberraciones cromosómicas (AC) e intercambio de cromátides hermanas (ICH).
Resultados. Se encontró diferencia significativa entre el promedio de ambos grupos para AC $(0.42$ \pm 0.23 vs $0.12 \pm 0.12, \mathrm{p}<0.0001)$ e $\mathrm{ICH}(4.17 \pm$ 0.82 vs $3.46 \pm 0.50, p<0.01)$. El análisis de correlación reveló diferencias significativas entre el \% de AC vs años de exposición $(\mathrm{p}=0.023)$ y entre el ICH y años de exposición $(p=0.037)$. La citometría hemática se encontró en límites normales para todos los individuos.

Comentarios. Las diferencias encontradas entre los dos grupos permite identificar un efecto citogenético temprano ocasionado por la exposición a rayos $X$, con incremento mayor correlacionado con la antigüedad de la exposición. Las manifestaciones citogenéticas fueron más tempranas que las hematológicas en este grupo estudiado. (Rev Biomed 2002; 13:76-82)

Palabras clave: Rayos $\mathrm{X}$, exposición laboral, aberraciones cromosómicas, intercambio de cromátides hermanas.

Solicitud de sobretiros: José M. Ceballos-Quintal, Laboratorio de Genética, Centro de Investigaciones Regionales "Dr. Hideyo Noguchi”, Universidad Autónoma de Yucatán, Calle 59 \# 490 x Av. Itzáes. C.P. 97000, Mérida, Yucatán, México. E-mail: jquintal@tunku.uady.mx Fax: (999) 9-23-61-20 Recibido el 12/Marzo/2001. Aceptado para publicación el 29/Junio/2001. 
JM Ceballos-Quintal, D Pinto-Escalante, J Canto-Herrera.

\section{SUMMARY.}

Increase in chromosomic aberrations and sister chromatid exchange in healthy individuals with laboral exposure to $X$ rays.

Introduction. Ionizing radiation produces different effects on humans, depending on the magnitude and source of exposure. One of these is laboral exposure to $\mathrm{X}$ rays, in which a chronic exposure of low doses may occur. Early identification of biological markers to recognize the risk of exposure result useful for the evaluation of high risk groups. In this study we analyze cytogenetic manifestations produced by labor exposure to diagnostic $\mathrm{X}$ rays, with the aim of identifying chromosomal changes in individuals exposed at different times and levels. Material and Methods. A group of individuals exposed to $\mathrm{X}$ rays and another of healthy unexposed individuals were included. We carried out blood count and chromosomal analysis: chromosomal aberrations (CA) and sister chromatid exchange (SCE), on all subjects.

Results. A significant difference was found between the two groups for CA $(0.42 \pm 0.23$ vs $0.12 \pm 0.12, \mathrm{p}<0.0001)$ and $\operatorname{SCE}(4.17 \pm 0.82$ vs $3.46 \pm 0.50, \mathrm{p}<0.01)$. Correlation analysis showed significant differences between CA \% vs. years of exposure $(p=0.023)$ and SCE vs. years of exposure $(p=0.037)$. Blood count was normal for all individuals.

Comments. The differences found between the two groups identified early chromosomal effect produced by X-ray exposure; which increased with time. Cytogenetic damage ocurred earlier than hematological damage in this group.

(Rev Biomed 2002; 13:76-82)

Key words: $X$ rays, laboral exposure, chromosomal aberrations, sister chromatid exchange.

\section{INTRODUCCIÓN.}

La exposición a radiaciones ionizantes produce diversos efectos en el hombre, entre los que se incluyen alteraciones hematológicas, reproductivas, neoplásicas y citogenéticas, entre otras (1). Los estudios sobre efectos a radiaciones se han extendido a otras formas de radiación: radiación ionizante, campos electromagnéticos, microondas, radionuclidos y ultrasonido (2-7). Actualmente se ha considerado la posibilidad de que las radiaciones emitidas por los teléfonos celulares sean también la explicación para ciertas neoplasias.

Habitualmente se puede identificar una fuente de exposición, ya sea accidental, laboral o por actividades rutinarias. Cuando la exposición es por algún accidente generalmente la exposición es aguda y la dosis de exposición es grande. Los efectos tempranos en los afectados pueden ser intensos, incluyendo la muerte, y a largo plazo puede ocurrir afecciones como neoplasias, trastornos en la función reproductiva o en la descendencia. En el otro extremo se encuentran las personas que desconocen estar expuestas a radiaciones debido a que las fuentes de exposición son generalmente fuentes de uso común. En estos casos los efectos se observan a largo plazo.

En las exposiciones a fuentes diversas de radiación por actividades laborales, suele conocerse el riesgo por la exposición y existir medidas encaminadas a la prevención de efectos adversos. Sin embargo, las disposiciones vislumbradas para la protección pueden ser inadecuadas o insuficientes, lo que da como resultado una exposición crónica a dosis pequeñas. Esta situación puede darse con desconocimiento de la persona expuesta, por lo que la identificación de marcadores biológicos de efecto temprano para el reconocimiento del riesgo de exposición resultan de utilidad para la evaluación de grupos en riesgo. Por este motivo, en este trabajo analizamos las manifestaciones citogenéticas de la exposición laboral a radiaciones ionizantes $\mathrm{X}$. Los rayos $\mathrm{X}$ (RX) usados para diagnóstico y terapia, son fotones de radiación electromagnética. Su energía es mayor que la de los rayos gamma y su origen es diferente (8-11). La secuencia de eventos ocurridos por la

\section{Revista Biomédica}


Manifestaciones cromosómicas por exposición a rayos $X$.

absorción de la radiación, abarca desde el nivel atómico de las moléculas afectadas hasta el nivel de la función celular. El tiempo de desarrollo de los cambios ocasionados por la radiación, varía desde $1 \times 10^{-16}$ segundos, hasta años o décadas. El efecto más importante de la radiación sobre las células es su inferencia con la reproducción celular. Como resultado, los tejidos que se reproducen con mayor velocidad, son los más afectados. Los efectos inmediatos de la exposición a la radiación son vistos con mayor frecuencia en piel y tejido sanguíneo $(9,11)$. La proporción, aparición, extensión y severidad de estos cambios depende de la energía, cantidad y tiempo de exposición recibida. La exposición fraccionada produce diferentes respuestas a la misma dosis total, es decir, que la aparición del daño podría ocurrir a medida que las dosis se acumulan (12).

El propósito de este trabajo es determinar los cambios cromosómicos presentes en individuos expuestos por diferentes períodos de tiempo y con diferentes grados de exposición a RX.

\section{MATERIAL Y MÉTODOS.}

La población estudiada quedó formada por dos grupos. Uno estuvo integrado por 25 sujetos expuestos a radiación ionizante $\mathrm{X}$ por laborar en un gabinete diagnóstico. Todos usaron pared blindada como protección, el uso de mandiles fue inconstante. El otro grupo estuvo constituido por 25 sujetos, principalmente profesores universitarios y empleados, no expuestos a radiación ni otros factores considerados de riesgo para producir manifestaciones cromosómicas, que participaron como controles, pareados por edad y sexo con los individuos expuestos. Cada individuo participó de forma voluntaria después de haber sido informados del motivo del estudio. Todas las personas incluidas otorgaron por escrito su consentimiento de participación y de uso de la información obtenida.

A cada sujeto se le realizó un interrogatorio directo para obtener datos generales (edad, género, estado de salud reproductivo), de exposición a factores de riesgo para la producción de manifestaciones cromosómicas (pesticidas, medicamentos antineoplásicos, alcohol, drogas psicotrópicas) y de exposición a RX (laboral u otra forma).

Los análisis realizados a todos los sujetos de ambos grupos fueron, citometría hemática completa y análisis cromosómico: aberraciones cromosómicas (AC) e intercambio de cromátides hermanas (ICH). Para su realización se obtuvo 7 $\mathrm{mL}$ de sangre venosa. La obtención de cromosomas se realizó por cultivo celular (72 h) de sangre total en medio RPMI-1640, adicionado con fitohemaglutinina para estimular el crecimiento celular. Adicionalmente se añadió desde el inicio de la incubación $0.04 \mathrm{~mL}$ de 5 ' Bromodeoxiuridina (5'BrdU, concentración de $500 \mu \mathrm{g} / \mathrm{mL}$ ) para obtener tinción diferencial de cromátides hermanas. Los cultivos se incubaron a $37^{\circ} \mathrm{C}$ por $72 \mathrm{~h}, 90^{\prime}$ antes de este período se agregó $0.5 \mathrm{~mL}$ de colchicina $(20 \mathrm{mg} / 100 \mathrm{~mL})$ para detener el ciclo celular en metafase. Al concluir la incubación, las células se sometieron a hipotonía con $\mathrm{KCl} 0.075$ $\mathrm{M}$, se fijaron con solución Carnoy (Metanol/ácido acético 3:1) y se prepararon en portaobjetos para tinción y análisis cromosómico.

El análisis de aberraciones cromosómicas se realizó en 100 metafases teñidas con colorante de Giemsa, que tiñe ambas cromátides de un color uniforme oscuro. Se buscaron alteraciones numéricas y estructurales (brechas, rupturas, fragmentos acéntricos fragmentos diminutos, anillos y figuras radiales) $(13,14)$.

Para la detección de ICH se analizaron 25 metafases con tinción diferencial de cromátides, realizado con colorante de Hoechst 33258 y Giemsa, en placas expuestas a luz UV por 120'. Para este análisis se contabilizó el número de rompimientos que participaron en el intercambio, de tal forma que para intercambios terminales se contabilizó un rompimiento y para los intersticiales fueron dos rompimientos. Se excluyeron de la cuenta los intercambios ocurridos a nivel de centrómero. Del número total de intercambios/ 
JM Ceballos-Quintal, D Pinto-Escalante, J Canto-Herrera.

metafase/individuo se obtuvo el promedio individual. De la suma de los promedios individuales de todos los integrantes de cada grupo obtuvimos la cifra del promedio de cada grupo para realizar el análisis estadístico.

De cada análisis citogenético se obtuvo el promedio y la desviación estándar individual y de grupo. Por medio de la t de student se analizó la significancia de los promedios entre los dos grupos. Para conocer la asociación entre los hallazgos citogenéticos y las variables de edad y tiempo de exposición se realizaron análisis de regresión lineal simple y múltiple. Todos los análisis estadísticos se efectuaron por medio de procedimientos disponibles en el paquete estadístico SPSS, versión 9.

\section{RESULTADOS.}

De los 25 sujetos de cada grupo, 5 fueron de

Cuadro 1

Características y resultados citogenéticos de los grupos expuesto a RX y control.

\begin{tabular}{|c|c|c|c|c|c|c|c|c|c|c|c|c|c|}
\hline \multirow[t]{2}{*}{ No } & \multicolumn{2}{|l|}{ Sexo } & Edad ${ }^{*}$ & \multicolumn{2}{|c|}{ Cigarros $^{\dagger}$} & \multicolumn{2}{|c|}{ Alcohol $^{\ddagger}$} & \multicolumn{2}{|c|}{$\begin{array}{c}\text { Pérdidas } \\
\text { gestacionales }\end{array}$} & \multicolumn{2}{|r|}{$\begin{array}{l}\mathrm{ICH}^{\S} \\
(\mathrm{XDS})\end{array}$} & \multicolumn{2}{|c|}{$\% \mathrm{AC}^{\|}$} \\
\hline & & $\mathrm{E}$ & $\mathrm{C}$ & $\mathrm{E}$ & $\mathrm{C}$ & E & $\mathrm{C}$ & $\mathrm{E}$ & $\mathrm{C}$ & E & $\mathrm{C}$ & E & $\mathrm{C}$ \\
\hline 01 & $\mathrm{M}$ & 36 & 38 & 0 & 0 & 0 & 0 & 0 & 0 & $3.48 \pm 1.71$ & $3.20 \pm 0.95$ & 0.3 & 0.1 \\
\hline 02 & $\mathrm{M}$ & 34 & 34 & 0 & 0 & 1 & 0 & 0 & 0 & $3.12 \pm 1.48$ & $3.96 \pm 1.51$ & 0.1 & 0 \\
\hline 03 & $\mathrm{M}$ & 25 & 25 & 6 & 2 & $<1$ & $<1$ & 0 & $1 \mathrm{ab}$ & $6.92 \pm 2.43$ & $3.92 \pm 1.52$ & 0.4 & 0.2 \\
\hline 04 & $\mathrm{M}$ & 35 & 35 & 0 & 0 & $<1$ & $<1$ & 0 & 0 & $4.24 \pm 1.96$ & $3.04 \pm 1.01$ & 0.3 & 0.1 \\
\hline 05 & $\mathrm{M}$ & 32 & 32 & 0 & 3 & 0 & $<1$ & 0 & 0 & $3.20 \pm 1.52$ & $3.72 \pm 1.56$ & 0.4 & 0 \\
\hline 06 & $\mathrm{M}$ & 35 & 35 & 0 & 0 & 0 & $<1$ & 0 & 0 & $4.24 \pm 1.71$ & $2.80 \pm 1.11$ & 0.1 & 0.4 \\
\hline 07 & $\mathrm{M}$ & 30 & 30 & 0 & 10 & 0 & $<1$ & 0 & 0 & $3.16 \pm 0.98$ & $3.12 \pm 0.97$ & 0.2 & 0.2 \\
\hline 08 & $\mathrm{~F}$ & 30 & 31 & 0 & 0 & 0 & 0 & 0 & 0 & $4.68 \pm 2.28$ & $3.44 \pm 1.22$ & 0.2 & 0.2 \\
\hline 09 & M & 34 & 34 & 5 & 0 & $<1$ & $<1$ & 0 & 0 & $5.04 \pm 1.83$ & $3.32 \pm 1.40$ & 0.7 & 0 \\
\hline 10 & $\mathrm{M}$ & 28 & 28 & 0 & 0 & $<1$ & $<1$ & 0 & 0 & $4.44 \pm 2.04$ & $3.68 \pm 0.98$ & 0.7 & 0.1 \\
\hline 11 & $\mathrm{M}$ & 42 & 43 & 1 & 0 & 1 & $<1$ & 0 & $1 \mathrm{ab}$ & $4.36 \pm 1.31$ & $4.04 \pm 1.85$ & 0.3 & 0 \\
\hline 12 & $\mathrm{~F}$ & 24 & 25 & $<1$ & 0 & $<1$ & $<1$ & 0 & $1 \mathrm{ab}$ & $3.80 \pm 1.47$ & $3.28 \pm 1.54$ & 0.6 & 0.4 \\
\hline 13 & $\mathrm{~F}$ & 42 & 43 & 6 & 0 & 1 & $<1$ & 0 & 0 & $4.68 \pm 1.88$ & $3.08 \pm 1.51$ & 0.1 & 0.2 \\
\hline 14 & $\mathrm{M}$ & 45 & 47 & 3 & 0 & $<1$ & $<1$ & 0 & 0 & $3.92 \pm 1.25$ & $3.00 \pm 1.38$ & 0.8 & 0 \\
\hline 15 & $\mathrm{M}$ & 35 & 37 & 0 & 5 & 1 & $<1$ & 0 & $3 a b$ & $4.00 \pm 1.47$ & $5.04 \pm 2.58$ & 0.5 & 0.2 \\
\hline 16 & M & 40 & 40 & 5 & 0 & 0 & $<1$ & 0 & 0 & $3.92 \pm 1.93$ & $3.72 \pm 1.56$ & 0.7 & 0 \\
\hline 17 & $\mathrm{M}$ & 48 & 47 & 0 & 0 & 0 & $<1$ & 0 & 0 & $3.56 \pm 1.41$ & $2.96 \pm 1.42$ & 0.7 & 0.2 \\
\hline 18 & M & 31 & 32 & $<1$ & 0 & $<1$ & $<1$ & 0 & 0 & $3.56 \pm 1.63$ & $3.56 \pm 1.29$ & 0.7 & 0 \\
\hline 19 & M & 35 & 34 & 0 & 0 & 0 & 0 & 0 & 0 & $5.24 \pm 1.94$ & $3.44 \pm 1.80$ & 0.2 & 0.1 \\
\hline 20 & M & 34 & 33 & 0 & & $<1$ & $<1$ & 0 & 0 & $4.48 \pm 2.12$ & $2.88 \pm 1.61$ & 0.7 & 0 \\
\hline 21 & $\mathrm{~F}$ & 35 & 32 & 0 & $<1$ & $<1$ & $<1$ & 0 & 0 & $3.64 \pm 1.43$ & $3.24 \pm 1.26$ & 0.5 & 0 \\
\hline 22 & $\mathrm{M}$ & 29 & 29 & 6 & 0 & $<1$ & $<1$ & 1 óbito & 0 & $3.28 \pm 1.27$ & $3.96 \pm 1.51$ & 0.4 & 0.2 \\
\hline 23 & $\mathrm{M}$ & 33 & 34 & 3 & 0 & $<1$ & $<1$ & 0 & 0 & $4.68 \pm 2.01$ & $2.96 \pm 1.36$ & 0.1 & 0.3 \\
\hline 24 & $\mathrm{M}$ & 30 & 32 & 0 & 0 & $<1$ & $<1$ & 0 & 0 & $4.20 \pm 1.44$ & $4.04 \pm 1.24$ & 0.4 & 0 \\
\hline 25 & $\mathrm{~F}$ & 36 & 38 & 0 & 5 & $<1$ & $<1$ & $1 \mathrm{ab}$ & $3 a b$ & $4.56 \pm 1.78$ & $3.20 \pm 1.35$ & 0.6 & 0.2 \\
\hline$P D^{\mathrm{II}}$ & & & & & & & & & & $4.17 \pm 0.82$ & $3.46 \pm 0.50$ & $0.42 \pm 0$ & $0.12 \pm 0.12$ \\
\hline $\mathrm{p}^{* *}$ & & & & & & & & & & & 0.01 & & 001 \\
\hline
\end{tabular}

$\mathrm{E}=$ expuesto, $\mathrm{C}=$ contol. * Edad en años, $†$ Número de cigarros por día, $\ddagger$ Vasos de bebidas alcohólicas por semana, $\S \mathrm{ICH}$, promedio $\pm \mathrm{DE} / 25$ células, $\|$ AC tipo cromátide y tipo cromosoma/100 células, II promedio \pm DS de cada grupo, ** t de student, gl 24.

En negrita se encuentran los resultados del grupo expuesto que son mayores al promedio encontrado en el grupo control.

\section{Revista Biomédica}




\section{Manifestaciones cromosómicas por exposición a rayos $X$.}

Cuadro 2

Análisis de regresión lineal simple y múltiple de las manifestaciones citogenéticas.

\begin{tabular}{lccc}
\hline Punto Final & $\mathrm{R}$ & $\mathrm{r}^{2}$ & $\mathrm{p}$ \\
\hline \% de AC vs. años de exposición & 0.321 & 0.103 & 0.023 \\
ICH vs. años de exposición & 0.296 & 0.088 & 0.037 \\
AC vs. edad casos & 0.015 & 0.124 & 0.554 \\
AC vs. edad controles & 0.066 & -0.256 & 0.216 \\
ICH vs. edad casos & 0.032 & -0.179 & 0.391 \\
ICH vs. edad controles & 0.059 & -0.243 & 0.243 \\
Edad vs. años de exp. Vs ICH & 0.363 & 0.131 & 0.036 \\
Edad vs. años de exp. vs AC & 0.327 & 0.107 & 0.022 \\
\hline
\end{tabular}

género femenino y los demás masculinos. El intervalo de edad del grupo expuesto (24-48) y control (25-47) fue similar (cuadro 1). De los factores de exposición analizados por su potencial para producir incremento en los análisis citogenéticos encontramos lo siguiente (cuadro 1):

Los sujetos que manifestaron ingerir bebidas alcohólicas lo hacían de forma eventual, en cantidad moderada, en ambos grupos.

Acerca del consumo de cigarros por individuo, la mayoría de los individuos de ambos grupos no fumaban o lo hacía en cantidades mínimas, excepto un control que manifestó fumar en forma moderada (10 cigarros/día).

La ingesta reciente (6 meses previos) de algún medicamento o droga psicotrópica fue negada por los sujetos de ambos grupos.

El intervalo de tiempo de trabajo con exposición a RX fue de 0.6 a 30 años, con un promedio de 7.9 (DE 6.7). El número de estudios radiográficos a los que cada trabajador estimó estar expuesto al día se encontró con un intervalo de 2 a 75, promedio de 35.76 y DE de 19.79 (cuadro 1).

Adicionalmente se presentan las pérdidas gestacionales manifestadas por cada sujeto, se encontró menor en el grupo expuesto que el control.

Se realizaron comparaciones de los promedios de ICH y AC entre los grupos expuesto y control, encontrándose diferencia significativa entre ambos grupos para los dos análisis citogenéticos (cuadro 1).
En el cuadro 2 se presentan los análisis de correlación realizados entre: \% de AC e ICH y años de exposición, edad de cada grupo y edad mas años de exposición. Se encontró diferencias significativas entre el \% de ICH y AC vs. años de exposición y en el análisis conjunto de ICH y AC vs. edad y años de exposición.

La citometría hemática de todos los sujetos estuvo en límites normales.

\section{DISCUSIÓN.}

Los diferentes efectos que produce la exposición a radiaciones ionizantes sigue siendo una inquietud, en particular para aquellos sujetos que quedan expuestos a dosis menores durante tiempo prolongado. La evaluación de estas personas resulta compleja, pues durante un período largo intervienen otros factores de exposición que pudieran modificar el efecto de exposición aislado a RX. En el grupo que estudiamos, las exposiciones ajenas a RX fueron mínimas, al menos para el período de 6 meses previo a la toma de sangre, por lo que los resultados encontrados en este trabajo podrían atribuirse a la exposición a radiaciones.

Uno de los factores principales relacionados con la exposición, es la dosis o cantidad de radiación recibida. Las radiaciones ionizantes son capaces de producir aberraciones en todas las etapas del ciclo celular, incluyendo aberraciones tipo cromosoma en G1 y aberraciones tipo cromátide en G2. Los linfocitos de sangre periférica, la subpoblación sanguínea que resulta estimulada en cultivo, se encuentran en general en la etapa de G0 (15). Por lo tanto, después de la exposición a radiaciones, pueden inducirse aberraciones tipo cromosoma en estas células, observables en metafases estimuladas en cultivo. La frecuencia se considera relacionada a la dosis recibida. Sin embargo, durante exposiciones por largos períodos de tiempo (meses o años), la frecuencia de aberraciones no es aditiva con el tiempo (o dosis), porque muchas aberraciones tipo 


\section{JM Ceballos-Quintal, D Pinto-Escalante, J Canto-Herrera.}

cromosoma son letales para la célula y son reemplazadas por células normales que derivan de células precursoras normales. La frecuencia de aberraciones cromosómicas parece ser constante por un período de seis semanas posteriores a la exposición $(1,16)$ Por otro lado, por el hecho de que algunos linfocitos tienen períodos de vida muy largos, significa que las aberraciones estables inducidas por radiaciones pueden identificarse mucho tiempo después de la exposición (9).

En los resultados que encontramos, el análisis de los promedios de cada grupo, mostró mayor número de $\mathrm{ICH}$ y $\mathrm{AC}$ en los sujetos expuestos, a pesar de las medidas de protección utilizadas, lo que podría estar indicando que se utilizan de manera incorrecta o son insuficientes. Por medio del análisis de correlación lineal simple y múltiple encontramos que hubo diferencia significativa entre el \% de AC e ICH y los años de exposición, es decir que existe incremento en los hallazgos citogenéticos cuando la antigüedad de la exposición es mayor. En donde no se encontró diferencias significativas fue al correlacionar la edad de los casos y de los controles vs las AC y los ICH. Esta falta de correlación nos permite interpretar que le edad de los sujetos no influyó significativamente en las manifestaciones citogenéticas. Estos hallazgos se corroboran al analizar estas dos variables juntas (la edad vs. los años de exposición y $\mathrm{AC}$ e ICH), que nos permiten interpretar que las diferencias que encontramos entre las AC y los ICH de cada grupo pueden ser atribuidas a la exposición a las radiaciones y que está en relación con los años de exposición pero no con la edad de los individuos. La diferencia de edad entre los sujetos de cada grupo no fue un factor que modificó las manifestaciones cromosómicas.

Se ha descrito que una de las manifestaciones iniciales por exposición a radiaciones es la presencia de anemia. En nuestro grupo este hallazgo no se comprobó, lo que podría interpretarse como que las manifestaciones citogenéticas son un evento aún más temprano que las hematológicas en los sujetos expuestos. Con estos hallazgos podría considerarse incluir a los estudios cromosómicos como un marcador de efecto temprano cuando se quiera evaluar una población expuesta a RX.

Resulta de importancia la detección de grupos expuestos a diferentes agentes que induzcan manifestaciones cromosómicas, pues éstas se han relacionado con la aparición de neoplasias en un tiempo variable después de la exposición (17-19). Para las personas expuestas laboralmente a radiaciones, un estudio de seguimiento a largo plazo (o un estudio retrospectivo de personas que estuvieron expuestas) nos permitiría saber si su exposición laboral está o no relacionada con la aparición de neoplasias y conocer el o los tejidos susceptibles.

\section{REFERENCIAS.}

1.- Cockerham LG, Mickley GA, Walden TL, Jr Stuart BO. Ionizing Radiation. En: Hayes W, editor. Principles and Methods of Toxicology. Third Edition. New York: Raven Press, Ltd; 1994. p.447-91.

2.- Graham JM Jr., Jones KL, Brent RL. Contribution of clinical teratologists and geneticists to the evaluation of the etiology of congenital malformations alleged to be caused by environmental agents: ionizing radiation, electromagnetic fields, microwaves, radionuclides, and ultrasound. Teratology 1999; 59:307-13.

3.- Brent RL Reproductive and teratologic effects of lowfrequency electromagnetic fields: a review of in vivo and in vitro studies using animal models. Teratology 1999; 59:261-86.

4.- Jensh RP, Brent RL. Intrauterine effects of ultrasound: animal studies. Teratology 1999; 59:240-51.

5.- Brent RL. Utilization of developmental basic science principles in the evaluation of reproductive risks from preand postconception environmental radiation exposures. Teratology 1999; 59:182-204.

6.- Yaguchi H, Yoshida M, Ding GR, Shingu K, Miyakoshi J. Increased chromatid-type chromosomal aberrations in mouse $\mathrm{m} 5 \mathrm{~S}$ cells exposed to power-line frequency magnetic fields. Int J Radiat Biol 2000; 76:1677-84.

7.- Vijayalaxmi, Leal BZ, Meltz ML, Pickard WF, Bisht

\section{Revista Biomédica}




\section{Manifestaciones cromosómicas por exposición a rayos $X$.}

KS, Roti JL, et al. Cytogenetic studies in human blood lymphocytes exposed in vitro to radiofrequency radiation at a cellular telephone frequency (835.62 MHz, FDMA). Radiat Res 2001; 155:113-21.

8.- Wang Y. Handbook of radiactive radionuclides. Cleveland: The Chemical Rubber Co.; 1969. p.837-67.

9.- Sandberg AA. The chromosomes in human cancer and leukemia. New York: Elsevier Science Publishing Co., Inc; 1983. p. 137-51.

10.- Gollnast HK, Vogel H. Radiation exposure in Computed Tomography. Aktuelle Radiol 1993; 3:20-3.

11. - Claus WD. Radiation Biology and Medicine. Massachusetts: Addison-Wesley, Reading; 1958. p 331.

12.- Marx MV, Niklason L, Mauger EA. Occupational radiation exposure to interventional radiologists: a prospective study. J Vasc Interv Radiol 1992; 3: 597-606.

13.- Evans HJ, O'Riordan ML. Human Peripheral blood lymphocytes for the analysis of chromosome aberrations in mutagen tests. En: Kilbey BJ, Legator M, Nichols W, Ramel C, editors. Handbook of mutagenicity test procedures. New York: Elsevier Scientific Publishing Company; 1977; p 261-74.

14.- Carrano AV, Natarajan AT. Considerations for population monitoring using cytogenetic techniques. Mut Res 1988; 204:379-406.

15.- Latt SA, Allen JW, Rogers WE, Juergens LA. In vitro and in vivo analysis of sister chromatid exchange formation. En: Kilbey BJ, Legator M, Nichols W, Ramel C editors. Handbook of mutagenicity test procedures. New York: Elsevier Scientific Publishing Company; 1977. p. 275-91.

16.- WHO. Guidelines for the study of genetic effects in human populations. Environmental health criteria 1985; 46: 41-3.

17.- Bonsái S, Abbondandolo A, Camurri L, Del Prá L, DeFerrari M, Degrassi F, et al. Are chromosome aberrations in circulating lymphocytes predictive of future cancer onset in humans?. Cancer Genet Cytogenet 1995; 79:133-5.

18.- Hagmar L, Brogegr A, Hansteen I, Heim S, Hoigsted B, Knudsen L, et al. Cancer risk in humans. Predicted by increased levels of chromosomal aberrations in lymphocytes: Nordic study group on the helth risk of chromosome damage. Cancer Res 1994; 54: 2919-92.
19.- Bonassi S, Hagmar L, Strömberg U, Huici M A, Tinnerberg H, Forni A, et al, and the European Study Group on Cytogenetic Biomarkers and Health (ESCH). Chromosomal aberrations in lymphocytes predict human cancer independently from exposure to carcinogens. Cancer Res. 2000; 60:1619-25. 